\title{
Predictors of poor glycemic control in type 2 diabetic patients attending public hospitals in Dar es Salaam
}

This article was published in the following Dove Press journal:

Drug, Healthcare and Patient Safety

24 October 2014

Number of times this article has been viewed

\author{
Appolinary R Kamuhabwa \\ Emmanuel Charles \\ Unit of Pharmacology and \\ Therapeutics, School of Pharmacy, \\ Muhimbili University of Health and \\ Allied Sciences, Dar es Salaam, \\ Tanzania
}

Correspondence: Appolinary R

Kamuhabwa

Unit of Pharmacology and Therapeutics, School of Pharmacy, Muhimbili University of Health and Allied Sciences,

PO Box 65013, Dar es Salaam, Tanzania

Tel +255757576985

Fax +255222 I50 465

Email enali2012@gmail.com or

akamuhabwa@muhas.ac.tz
Background: Tanzania has recently experienced a significant rise in the burden of diabetes, and it is estimated that more than 400,000 people are living with diabetes. A major concern in the management of diabetes is the occurrence of diabetic complications that occur as a result of poor glycemic control. Identification of the factors associated with poor glycemic control is important in order to institute appropriate interventions for the purpose of improving glycemic control and prevention of chronic complications.

Aim: The aim of this study was to determine the level of glycemic control and explore the factors associated with poor glycemic control among patients with type 2 diabetes mellitus (T2DM).

Methodology: A cross-sectional study was carried out at the diabetic clinics for T2DM patients at the national and municipal hospitals in Dar es Salaam. A total of 469 patients were enrolled over a period of 8 weeks from March 2013 to May 2013. Patients' information such as sociodemographic characteristics, self-care management behaviors, and medication adherence were obtained through interviews. Blood pressure, weight, and height were measured during the day of the interview. All available last readings for fasting blood glucose (FBG) measurements, lipid profile, and other clinical characteristics were obtained from patients' records.

Results: The mean age of patients was 54.93 years. The majority $(63.5 \%)$ of patients were females and only eight patients had records of lipid profile measurements. Out of 469 patients, $69.7 \%$ had FBG of $\geq 7.2 \mathrm{mmol} / \mathrm{L}$, indicating poor glycemic control. Females aged between 40 years and 59 years had significantly higher poor glycemic control (76.1\%) as compared with their male counterparts. Thirty-eight percent of patients had poor medication adherence, which was associated with poor glycemic control. The proportion of poor glycemic control increased with age. A significantly high proportion of poor glycemic control was observed in patients who had had the disease for more than 20 years since diagnosis. Factors associated with poor glycemic control included lack of health insurance, using more than one oral hypoglycemic agent, normal body mass index, obesity, and nonadherence to diabetic medications.

Conclusion: Patients in this study had generally poor glycemic control. From these findings it is recommended that diabetic patients should be routinely screened for lipid profile to determine levels of cholesterol, triglycerides, and low-density lipoproteins, which are risk factors for cardiovascular events. An education program should be developed to educate patients on the importance of medication adherence and weight management for better glycemic control.

Keywords: type 2 diabetes, lipid profile, self-management behaviors

\section{Introduction}

Tanzania has recently been experiencing a significant rise in the burden of diabetes. In the $1980 \mathrm{~s}$, the prevalence of type 2 diabetes mellitus (T2DM) was $0.9 \%$ in rural areas. ${ }^{1}$ In 2000 , the prevalence of diabetes was reported to be $1.3 \%$ in rural areas and 
$4.0 \%$ in urban populations. ${ }^{2}$ At present, the Tanzania Diabetes Association estimates that there are more than 400,000 people with diabetes in the country. In Tanzania, about \$4 million would have been required to take care of all the patients with diabetes in 1989-90, which translates to $\$ 138$ per patient per year. This sum is equivalent to $8.1 \%$ of the total budgeted health care expenditure for that financial year and is well above the allocated per capita health expenditure of $\$ 2$ for the year 1989-90. ${ }^{3}$

A major concern in the management of diabetes is the occurrence of complications, many of which are irreversible. Complications in diabetes occur as a result of the injurious effects of hyperglycemia. ${ }^{4}$ Long-term complications of diabetes can be broadly classified into two major categories: macrovascular (eg, peripheral arterial disease, stroke, and coronary artery disease) and microvascular (eg, retinopathy, neuropathy, and nephropathy). ${ }^{4}$ Other complications experienced by diabetic patients include skin complications, gastroparesis, dental infections, and depression. ${ }^{5}$ Common cardiovascular disease (CVD) events experienced by patients with diabetes include coronary heart diseases, myocardial infarction, stroke, cerebrovascular diseases, erectile dysfunction, and peripheral vascular diseases. ${ }^{4-6}$

For optimal management of people with diabetes, it is important that the health care team devise a treatment plan tailored specifically to the needs of the individual patient. The treatment plan should include adequate glycemic control (ie, self-monitoring of blood glucose levels and measurement of glycated hemoglobin $\left[\mathrm{HbA}_{1 \mathrm{c}}\right]$ ) as well as adequate blood pressure and blood lipid level monitoring. ${ }^{7}$ A treatment plan also requires dietary modifications, exercise, and administration of medication on schedule. Patients should be allowed to play active roles in the management of their health and encouraged to participate in diabetes self-management education programs. Studies have emphasized the importance of achieving optimal glucose control through strict adherence to medications, diet, and exercise in order to minimize serious long-term complications. ${ }^{7-9}$ Other important factors considered when developing a comprehensive treatment plan include the patient's age, eating behaviors, work or school schedule, sociocultural/socioeconomic factors, and presence of complications of diabetes or other comorbid conditions. $^{10}$

In the management of diabetes, it is important to provide adequate patient education on the disease state, associated complications, risk for developing the complications (including the signs and the symptoms), and importance of achieving and maintaining adequate glycemic control, especially as it relates to the prevention and management of diabetic complications. ${ }^{11}$ Diabetes management can be broadly classified into nonpharmacological (ie, lifestyle changes) and pharmacological (ie, drugs) interventions. Pharmacological intervention becomes necessary only when optimal glycemic control cannot be achieved through lifestyle changes alone. Generally, patients with an $\mathrm{HbA}_{1 \mathrm{c}}$ level $>7 \%$ or who present with symptoms can be managed using both pharmacological and nonpharmacological interventions.

Glycemic control is considered as the main therapeutic goal for prevention of organ damage and other complications of diabetes. Several large clinical trials ${ }^{12}$ have demonstrated that tight blood glucose control correlates with reduction of microvascular complications of diabetes. Therefore, achieving glycemic control is a critical metabolic goal because hyperglycemia contributes to the progression of diabetes mellitus by affecting both $\beta$-cell function and insulin sensitivity. ${ }^{13}$

In clinical practice, optimal control is difficult to obtain on a long-term basis because the reasons for poor glycemic control in T2DM patients are complex. Both patients and health care provider-related factors may contribute to poor glycemic control. In view of the benefits of strict glycemic control, several studies have been carried out to explore the variables that may be associated with poor control. ${ }^{14-16}$ Most of these studies, however, are from Europe and North America, with minimal data available from Asia and the African continent. A variety of factors are identified in influencing glycemic control, including age, sex, education, body mass index (BMI), smoking, duration of the disease, and type of medication.

Prospective randomized clinical trials and epidemiological studies have demonstrated that glycemic control is associated with reduced rates of retinopathy, nephropathy, neuropathy, and CVD. ${ }^{13}$ Despite the evidence from large randomized controlled trials establishing the benefit of intensive diabetes management in reducing microvascular and macrovascular complications, ${ }^{12}$ a high proportion of patients remain poorly controlled. Poor and inadequate glycemic control among patients with T2DM constitutes a major public health problem and is a major risk factor for the development of diabetes complications. Therefore, glycemic control remains the major therapeutic objective for prevention of target organ damage and other complications arising from diabetes.

Tanzania is a resource-limited setting where the majority of people are estimated to live below the poverty level, so economic access to antidiabetic medications and preventive 
measures may be restricted by the cost. Also, additional costs for treatment of comorbid conditions such as hypertension could have an impact on disease outcome. To our knowledge, no studies have been done in Tanzania to describe the risk factors associated with poor glycemic control in T2DM patients. However, studies from different parts of the world have found a variety of risk factors that predict poor glycemic control. Most of these studies were done in North America and Europe. This study focused on several factors and their association with glycemic control. It was designed to assess whether similar variables reported in other countries also play a role in Tanzania. Specifically, this study was done to identify the factors associated with poor glycemic control in T2DM patients in urban areas in Tanzania.

\section{Materials and methods Study design}

This was a cross-sectional study in which T2DM patients were sampled through systematic sampling in the public hospitals in Dar es Salaam. Data were obtained using questionnaires to assess responses from the patients on self-management behaviors, and sociodemographic and clinical characteristics. Moreover, data including biochemical parameters were extracted from patients' medical records.

\section{Study setting}

The study was carried out at Muhimbili National Hospital $(\mathrm{MNH})$ and all the three municipal hospitals in Dar es Salaam. These hospitals are Temeke, Amana, and Mwananyamala. $\mathrm{MNH}$ is the referral hospital and a university teaching hospital located in the Dar es Salaam region. The services that are provided in this hospital include management of diabetes in the endocrinology unit. Diabetic clinics are conducted twice a week and about 60 patients attend per day.

Temeke hospital is located in the Temeke district in Dar es Salaam. Diabetic clinics are conducted three times a week and about 35 patients attend per day. Amana hospital is located in the Ilala district and diabetes clinics are conducted twice per week with about 20 patients per clinic. Mwananyamala hospital, on the other hand, is located in the Kinondoni district. The clinics at Mwananyamala hospital are conducted three times per week with about 15 patients attending the clinic per day.

\section{Study population}

The target population was all T2DM patients aged $\geq 18$ years. These were patients attending diabetes clinics at $\mathrm{MNH}$, Temeke, Amana, and Mwananyamala hospitals. Apart from age, other inclusion criteria were patients who had had T2DM for $>1$ year and who had been on treatment for $\geq 3$ months. Very sick patients, newly diagnosed patients, pregnant women, and patients with mental disorders were excluded from the study. Using these criteria, a total of 498 patients were enrolled in the study from March 2013 to May 2013.

\section{Sampling technique}

A systematic sampling technique was used to recruit study participants. The target was to recruit 120 patients at $\mathrm{MNH}$, 160 at Temeke, 80 at Amana, and 90 at Mwananyamala hospitals depending on the average number of patients attending the clinic during the study period. Study participants were recruited consecutively. In every clinic, the first patient to be recruited into the study was selected by the nurse, and then every fourth patient was recruited into the study. This was done at Temeke, Mwananyamala, and Amana hospitals. At $\mathrm{MNH}$, patients were approached at the waiting area. The first patient was selected according to seat arrangement, and consecutive participants were selected after every fourth patient until the end of that day. The purpose of the study was explained to them and they were asked to participate in the study. Those who agreed to participate were interviewed using structured questionnaires while waiting to see the physician. Thereafter, information on fasting blood glucose (FBG), lipid profile, height, weight, blood pressure, coexisting diseases, and drugs used by the patients were recorded from patient files.

\section{Data collection}

Data were collected using structured questionnaires and designed forms. The main components of the questionnaire were the sociodemographic and background information, self-management behaviors, and medication adherence among T2DM patients. The data collection form was used to collect data on the laboratory investigations and measurements, type of medications, and coexisting diseases. This information was obtained from the patient files.

\section{Sociodemographic and self-management behaviors} Sociodemographic and background information included age of the patient, sex, marital status, employment status, level of education, height, weight, BMI, blood pressure, FBG, duration of the disease, and age of the patient at diagnosis. Data on self-management behaviors included diet, exercise, and self-monitoring of blood glucose. These were collected to assess the adherence to diabetic control measures that included physical exercise, diet, and blood glucose testing. 
Self-management behaviors were assessed using the Summary of Diabetes Self-Care Activities Measure scale, ${ }^{17}$ which contains eleven question items designed to ask the patients about their diabetes self-care activities during the past 7 days. If patients were sick during the past 7 days, they were asked to think back to the last 7 days that they were not sick.

\section{Medication adherence}

This was determined by self-reporting with the use of the eight-item Morisky scale. ${ }^{18}$ The scale contains questions asking the patient to respond "yes" or "no" to a set of eight questions. A positive response indicates a problem with adherence. Therefore, higher scores indicate that a patient is least adherent to medications. In this study, a positive response was awarded one point and a negative response was awarded zero points. Patients were classified as highly adherent (score of 0 ), moderately adherent (score of 1-2), and least adherent (score of 3-8).

\section{Laboratory investigations and measurements}

The available last readings of FBG measurement and lipid profile (high-density lipoproteins [HDL], low-density lipoproteins [LDL], triglycerides [TG], and cholesterol) were obtained from the patient files. These were the readings from 3 months to 1 year of treatment. According to the records, FBG was measured after obtaining finger prick blood samples from patients. Analysis was done using Accutrend ${ }^{\circledR}$ Plus machines (Roche, Mannheim, Germany).

During the day of the study, blood pressure was measured by a trained nurse. Weights were measured using a Seca scale with the patient wearing light clothes with no shoes. Weight was measured up to the nearest $100 \mathrm{~g}$. Heights were measured using a standard height board with the participant wearing no shoes. Measurements for height were then taken to the nearest $1 \mathrm{~cm}$. BMI was calculated as weight in $\mathrm{kg}$ divided by height in meters squared. BMI was categorized as normal $\left(<25 \mathrm{~kg} / \mathrm{m}^{2}\right)$, overweight $\left(25-29.99 \mathrm{~kg} / \mathrm{m}^{2}\right)$, and obese $\left(\geq 30 \mathrm{~kg} / \mathrm{m}^{2}\right)$. Hypertension was defined as a patient with systolic/ diastolic pressure $>140 / 90 \mathrm{mmHg}$ or those already using hypertensive drugs. Glycemic status was categorized as good glycemic control if FBG was $\leq 130 \mathrm{mg} / \mathrm{dL}$ (7.2 $\mathrm{mmol} / \mathrm{L})$ and poor control if $\mathrm{FBG}$ was $>130 \mathrm{mg} / \mathrm{dL}$ (7.2 mmol/L). Assessment for abnormal lipid profiles was done as described by the American Diabetes Association. ${ }^{19}$ Dyslipidemia was defined as a patient with one or more of the abnormalities for serum lipids or the patient receiving medication for management of dyslipidemia.

\section{Data analysis}

Data were analyzed using Statistical Package for Social Sciences software, version 20 (IBM Corporation, Armonk, NY, USA). Patients' sociodemographic characteristics and diabetes-specific variables were summarized using frequency distribution tables. Data were described using means for continuous variables (age, duration of disease, systolic blood pressure, and diastolic blood pressure) and proportions for categorical variables (education level, sex, dyslipidemia, FBG, cholesterol level, HDL, LDL, triglycerides, occupation, and type of treatment). Associations between variables were tested by the use of the chi-square test. All factors with a $P$-value $\leq 0.2$ were considered for multivariate logistic regression to determine independent factors predicting poor glycemic control.

The dependent variables were described as a dichotomous variable that categorized $\mathrm{FBG}$ of $\leq 7.2 \mathrm{mmol} / \mathrm{L}$ as good glycemic control and $\mathrm{FBG}$ of $>7.2 \mathrm{mmol} / \mathrm{L}$ as poor glycemic control. The independent variables were duration of the disease since diagnosis, age at onset, age of the patient, BMI (overweight vs normal weight/obesity), health insurance, oral hypoglycemic agents (OHAs) being used (monotherapy vs combination therapy), and medication adherence (high adherence vs low/medium adherence). A probability $(P)$ value $\leq 0.05$ was considered statistically significant.

\section{Ethical consideration}

Ethical clearance for the study was sought from the Muhimbili University of Health and Allied Sciences Ethical Committee. Permission to conduct the study at $\mathrm{MNH}$ was sought from the office of the executive director. Permission to conduct the study in the municipal hospitals was sought from the respective district medical officers in charge. The aim and objectives of the study were explained to the respondents. Before conducting interviews, the willingness of respondents was required and written informed consent was provided. All data collected during the study were treated with strict confidentiality. Questionnaires and data collection forms were assigned study numbers, and names of the patients were not used during data collection or entered into the computer for analysis.

\section{Results}

During the 8-week study period, 498 T2DM patients were recruited to participate in the study. Sixteen patients did not finish the questionnaires and 13 patients who did not have FBG results were excluded from the final data analysis. Therefore, a total of 469 patients provided the required information for the study. 


\section{Sociodemographic characteristics of T2DM patients}

Most (63.5\%) of the patients were females. Females had a mean age of $53.8 \pm 11.51$ years as compared with males with a mean age of $56.8 \pm 12.44$ years. The age of study participants ranged from 25 years to 85 years with an overall mean age of $54.94 \pm 11.93$ years. The majority $(89.3 \%)$ of patients were aged $>40$ years. About half $(51.4 \%)$ of the patients had attained primary school education, 331 (70.6\%) were married, 368 (69.9\%) were unemployed, and only 101 $(21.5 \%)$ had health insurance coverage. The sociodemographic factors of the study participants are summarized in Table 1.

\section{Association of sociodemographic factors and poor glycemic control}

In comparison with other health facilities, Amana hospital had a significantly higher percentage of patients with poor glycemic control $(80.5 \%)(P=0.015)$. The proportions of patients with poor glycemic control in the other hospitals were $67.6 \%$ at Mwananyamala and $61.2 \%$ at $\mathrm{MNH}$.

Analysis for the association between sociodemographic factors of the patients and poor glycemic control showed that the proportion of patients with poor glycemic control

Table I Sociodemographic characteristics of type 2 diabetes mellitus patients who were recruited in the study $(n=469)$

\begin{tabular}{lll}
\hline Characteristics & Number & Percentage \\
\hline $\begin{array}{l}\text { Age (years) } \\
\text { 18-39 }\end{array}$ & 50 & 10.7 \\
$40-59$ & 241 & 51.3 \\
$\geq 60$ & 178 & 38 \\
Sex & & \\
Male & 171 & 36.5 \\
Female & 298 & 63.5 \\
Education level & & \\
No formal education & 139 & 29.6 \\
Primary school & 241 & 51.4 \\
Secondary school & 76 & 16.2 \\
Tertiary school & 13 & 2.8 \\
Marital status & & \\
Married & 331 & 70.6 \\
Single & 20 & 4.3 \\
Widow/widower & 91 & 19.4 \\
Divorced & 27 & 5.8 \\
Employment status & & 12.6 \\
Employed & 59 & 69.9 \\
Not employed & 328 & 17.5 \\
Self-employed & 82 & 21.5 \\
Health insurance & & 78.8 \\
Insured & 101 & \\
Not insured & 368 & \\
\hline
\end{tabular}

was $72.6 \%$ in the age group of $40-59$ years but was relatively higher than those in the other age groups $(P=0.19)$. Diabetes was more likely to be poorly controlled in patients who did not have health insurance $(71.7 \%)$ as compared with those who were insured for health care services $(62.4 \%)$ $(P=0.007)$. Marital status, sex, employment, and education level of the patients were not significantly associated with glycemic control.

Stratification by age and sex showed that females aged 40-59 years had a significantly higher percentage of poor glycemic control than their male counterparts of the same age group ( $76.1 \%$ vs $65.4 \%, P=0.04$ ). Also, females aged 40-59 years had significantly higher BMI (obese) than their male counterparts of the same age group (35.4\% vs $11.5 \%$, $P=0.0000)$.

\section{Clinical characteristics of T2DM patients}

Three hundred and thirty (70.4\%) patients had confirmed T2DM for $<10$ years. The overall mean (standard deviation) duration of the disease was $7.19 \pm 6.04$ years with a minimum of 1 year and a maximum of 33 years. Most $(75.3 \%)$ of the patients were diagnosed with T2DM when they were aged $>40$ years. Among these patients, the mean FBG was $10.3 \pm 4.6 \mathrm{mmol} / \mathrm{L}$ and the proportion of patients with poor glycemic control was $69.7 \%$. The mean blood pressure for these patients was $138.07 / 85.18 \mathrm{mmHg}$. The mean BMI of T2DM patients in this study was $27.06 \pm 5.34$. Only eight out of 469 T2DM patients had records of their lipid profile measurements (Table 2).

Out of 469 T2DM patients, 327 (69.7\%) were poorly controlled with FBG $>7.2 \mathrm{mmol} / \mathrm{L}$. The proportion of poor glycemic control was found to increase with the duration of the disease, and it was significantly higher in patients who had longer duration of diabetes of $>20$ years than those who had the disease for $<10$ years $(P=0.027)$. Age at diagnosis and systolic and diastolic blood pressure were not significantly associated with glycemic control $(P>0.05)$. Patients with normal BMI (76.7\%) had a significantly higher percentage of poor glycemic control than overweight $(69.6 \%)$ and obese patients $(61.6 \%)(P=0.01)$. The results of association of clinical characteristics and poor glycemic control are summarized in Table 3.

\section{Type of comorbidities and antidiabetic drugs prescribed to T2DM patients}

Table 4 shows the summary of the comorbidities and antidiabetic drugs that were prescribed to the patients. The most frequent comorbidity was hypertension $(50.7 \%)$, followed 
Table 2 Association between clinical characteristics of type 2 diabetes mellitus patients and glycemic control $(n=469)$

\begin{tabular}{|c|c|c|c|}
\hline Variable & Number & $\begin{array}{l}\text { Poor glycemic } \\
\text { control (\%) }\end{array}$ & $P$-value \\
\hline \multicolumn{4}{|c|}{ Age at diagnosis (years) } \\
\hline $18-39$ & 116 & $81(69.8)$ & \\
\hline $40-59$ & 266 & $192(72.2)$ & 0.204 \\
\hline$\geq 60$ & 87 & $54(61.20)$ & \\
\hline \multicolumn{4}{|c|}{ Duration of disease (years) } \\
\hline $1-9$ & 330 & $219(66.4)$ & \\
\hline $10-19$ & 110 & $83(75.5)$ & 0.027 \\
\hline$\geq 20$ & 29 & $25(86.2)$ & \\
\hline \multicolumn{4}{|c|}{ Blood pressure $(\mathrm{mmHg})$} \\
\hline $\begin{array}{l}\text { Systolic blood } \\
\text { pressure } \leq 140\end{array}$ & 230 & $163(70.9)$ & 0.596 \\
\hline $\begin{array}{l}\text { Systolic blood } \\
\text { pressure }>140\end{array}$ & 239 & $164(68.6)$ & \\
\hline $\begin{array}{l}\text { Diastolic blood } \\
\text { pressure } \leq 90\end{array}$ & 274 & $193(70.4)$ & 0.689 \\
\hline $\begin{array}{l}\text { diastolic blood } \\
\text { pressure }>90\end{array}$ & 195 & I 34 (68.7) & \\
\hline \multicolumn{4}{|c|}{ Body mass index $\left(\mathrm{kg} / \mathrm{m}^{2}\right)$} \\
\hline$<25$ & 189 & I 45 (76.7) & \\
\hline $25-29.99$ & $|5|$ & $93(61.6)$ & 0.01 \\
\hline$\geq 30$ & 125 & 87 (69.6) & \\
\hline
\end{tabular}

by coronary heart disease (13.4\%). Osteoarthritis was observed in $56(11.9 \%)$ patients, while $8.5 \%$ of the patients had peptic ulcers. Other diseases such as depression, human immunodeficiency virus/acquired immunodeficiency syndrome, asthma, tuberculosis, rheumatism, and gout, with individual frequency $<3 \%$, all constituted $9.4 \%$ of the total comorbidities.

OHAs were prescribed to 391 (83.4\%) patients, while a combination of OHA and insulin was prescribed in only $2.1 \%$ of the patients. Insulin alone was prescribed to only $14.5 \%$ of the patients. Of the patients on OHAs, 247 (63.7\%) were on combination therapy, while $142(36.3 \%)$ were using monotherapies. The most prescribed combination therapy contained metformin and sulfonylureas (95.2\%). Of the patients on monotherapy with an OHA, 100 (65.2\%) patients were using metformin. The most common sulfonylureas prescribed to diabetic patients were glibenclamide and gliclazide. Other sulfonylureas prescribed to T2DM patients were chlorpropamide, glimepiride, and gliclazide.

Number of comorbidities, hypertension, and treatment modalities were not associated with poor glycemic control $(P>0.05)$. However, patients who were using combination therapy of OHAs had a very high proportion of poor glycemic
Table 3 Comorbidities and types of antidiabetic drugs prescribed to type 2 diabetes mellitus patients

\begin{tabular}{|c|c|c|}
\hline Characteristics & Number & $\%$ \\
\hline \multicolumn{3}{|l|}{ Comorbidities $(n=469)$} \\
\hline Hypertension & 238 & 50.7 \\
\hline Osteoarthritis & 56 & 11.9 \\
\hline Coronary heart disease & 63 & 13.4 \\
\hline Chronic heart failure & 17 & 3.6 \\
\hline Peptic ulcers & 40 & 8.5 \\
\hline Skin infection & 19 & 4.1 \\
\hline Others & 44 & 9.4 \\
\hline \multicolumn{3}{|l|}{ Antidiabetics $(n=469)$} \\
\hline $\mathrm{OHA}$ alone & 391 & 83.4 \\
\hline Insulin alone & 68 & 14.5 \\
\hline $\mathrm{OHA}$ and insulin & 10 & 2.1 \\
\hline \multicolumn{3}{|l|}{$\mathrm{OHA}(\mathrm{n}=39 \mathrm{I})$} \\
\hline Combination therapy & 249 & 63.7 \\
\hline Monotherapy & 142 & 36.3 \\
\hline \multicolumn{3}{|l|}{ OHA combination therapy $(n=249)$} \\
\hline Metformin and sulfonylureas & 237 & 95.2 \\
\hline Metformin and pioglitazone & 6 & 2.4 \\
\hline Sulfonylureas and pioglitazone & 1 & 0.4 \\
\hline Metformin, sulfonylureas, and pioglitazone & 5 & 2.0 \\
\hline \multicolumn{3}{|l|}{ OHA monotherapy $(n=\mid 42)$} \\
\hline Metformin & 92 & 64.8 \\
\hline Sulfonylureas & 44 & 31.0 \\
\hline Pioglitazone & 6 & 4.2 \\
\hline
\end{tabular}

Abbreviation: OHA, oral hypoglycemic agent.

control (73.5\%), compared with $60.6 \%$ of patients using monotherapies $(P=0.008)$.

\section{Self-management behaviors in T2DM patients}

One hundred and twenty patients (31.2\%) did not follow healthy eating plans. More than half of the patients (53\%) reported that they were advised by health care providers to take five or more servings of vegetables and fruit, while $18.6 \%$ of the patients indicated that they were not advised on meal intake. Most patients (63.3\%) were eating three servings per day. Only 90 (19.2\%) patients reported eating five or more servings of fruit and vegetables on a regular basis. Eighty patients (17.1\%) were eating fatty food such as red meat and full-fat dairy products. Three hundred and sixty-one (77\%) patients did not participate in physical activity and only a third (33\%) of patients were doing regular exercise. Of 469 patients, only 115 (24.5\%) of them tested their blood glucose at home. Out of those who tested their blood glucose at home, only $16(13.9 \%)$ patients monitored their blood glucose regularly. Sixty (52.2\%) patients reported that they were not advised by health care providers on how frequently they were required to monitor their blood glucose at home. 
Table 4 Association between self-management behavior and glycemic control among type 2 diabetes mellitus patients $(n=469)$

\begin{tabular}{|c|c|c|c|}
\hline Variable & Number & $\begin{array}{l}\text { Poor glycemic } \\
\text { control }\end{array}$ & $P$-value \\
\hline \multicolumn{4}{|l|}{ General diet $(n=385)^{a}$} \\
\hline$\geq 3$ days & 265 & I $82(68.7)$ & 0.795 \\
\hline$<3$ days & 120 & $84(70.0)$ & \\
\hline \multicolumn{4}{|l|}{ Average serving per day } \\
\hline$<3$ & 60 & & \\
\hline 3 servings & 297 & & \\
\hline$>3$ & 96 & & \\
\hline \multicolumn{4}{|c|}{$\geq 5$ servings of fruit and vegetables } \\
\hline$\geq 3$ days & 90 & $62(68.9)$ & 0.848 \\
\hline$<3$ days & 379 & $265(69.9)$ & \\
\hline \multicolumn{4}{|l|}{ High-fat foods } \\
\hline$\geq 3$ days & 80 & $65(68.8)$ & 0.835 \\
\hline$<3$ days & 389 & $272(69.9)$ & \\
\hline \multicolumn{4}{|l|}{ Physical activity } \\
\hline$\geq 3$ days & 108 & $79(73.1)$ & 0.377 \\
\hline$<3$ days & 361 & $248(68.7)$ & \\
\hline \multicolumn{4}{|l|}{ Specific exercise session } \\
\hline$\geq 3$ days & 155 & $105(67.7)$ & 0.525 \\
\hline$<3$ days & 314 & $248(70.7)$ & \\
\hline \multicolumn{4}{|c|}{ Self-monitoring of blood glucose } \\
\hline$\geq 5$ days & 16 & I3 (8I.2) & 0.425 \\
\hline$<5$ days & 99 & 71 ( 7I.9) & \\
\hline \multicolumn{4}{|l|}{ Medication adherence } \\
\hline Low adherence & 178 & $144(80.9)$ & \\
\hline Moderate adherence & 209 & $129(61.7)$ & 0.000 \\
\hline Good adherence & 82 & $54(65.9)$ & \\
\hline
\end{tabular}

Note: ${ }^{2} 4$ patients did not respond on a general diet.

\section{Medication adherence among T2DM patients}

Assessment of patients' responses to the eight-item modified Morisky adherence predictor scale showed that $17.5 \%$ of patients were highly adherent to the prescribed medications, $218(44.6 \%)$ patients were moderately adherent, while $38 \%$ of the patients were judged to have low adherence to the prescribed antidiabetic medications (Figure 1). The scale

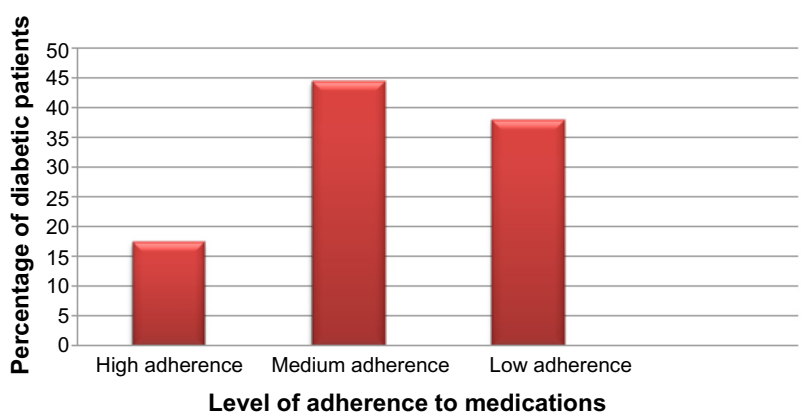

Figure I Levels of adherence to antidiabetic medications among type 2 diabetes mellitus patients $(n=469)$. showed that most $(57.1 \%)$ patients did miss taking their medications due to different reasons. The reported reasons for nonadherence were the cost of the medications $(39.5 \%)$, omission (18\%), unavailability of medications in the health facilities (16.9\%), travel (16.9\%), side effects (9.4\%), sense of feeling better $(4.1 \%)$, and use of alternative medicines $(2.6 \%)$. One hundred and sixty-two $(34.5 \%)$ patients reported that when symptoms were under control they stopped taking their medications.

There were no statistically significant differences between glycemic control and following a healthy eating plan, physical activity, and self-monitoring of blood glucose $(P>0.05)$. However, a relatively high proportion of poor glycemic control was observed in patients who did not follow health plans or do regular exercise and those who regularly monitored their blood glucose. The number of servings per day was also not associated with poor glycemic control. The proportion of patients with poor glycemic control was significantly higher in patients who were least adherent to antidiabetic medications (80.9\%) than patients who had moderate $(61.7 \%)$ and high medication adherence $(60.5 \%)(P=0.000)$. The results of the association between self-management behavior and glycemic control among T2DM patients are summarized in Table 4.

In order to control confounding factors, all factors with a $P$-value $\leq 0.2$ on univariate analysis were considered for multivariate analysis. Binary logistic regression analysis indicated that normal BMI, obesity, low medication adherence, combination therapy of OHAs, and lack of health insurance were the predictors of poor glycemic control. Other factors such as duration of the disease, age of patient, and age at diagnosis did not show statistical significance for association with glycemic control. It was further observed that poor glycemic control is 2.2 times more likely to occur in patients with normal BMI (odds ratio [OR] [95\% confidence interval $\{C I\}]=2.234[1.278,3.904])$ as compared with patients who were overweight. In addition, it was found that obese patients were 2.4 times more likely to have poor glycemic control (OR $[95 \% \mathrm{CI}]=2.347[1.274-4.324], P=0.006)$ than overweight patients.

Patients who were using combination therapy of OHAs were more than 2.5 times more likely to be poorly controlled (OR [95\% CI] =2.528 [1.475-4.331]) compared with patients who were on monotherapy. Results further showed that poor glycemic control is likely to occur 2.1 times more in patients with low medication adherence as compared with patients who were highly adherent to medications (OR [95\% $\mathrm{CI}]=2.084[1.069,4.060])$. Patients who did not have health 
insurance were more than 1.9 times more likely to have poor glycemic control compared with patients who were insured for health care $(\mathrm{OR}$ [95\% CI] $=1.861$ [1.044, 3.318]).

\section{Discussion}

The recommended FBG in diabetic patients should be $<7.2 \mathrm{mmol} / \mathrm{L}$, since values higher than this are associated with risks of microvascular complications. ${ }^{20}$ In this study, the majority of patients with T2DM had FBG levels $>7.2 \mathrm{mmol} / \mathrm{L}$. Hb1 Ac is an established gold standard in assessing glycemic control. The American Diabetes Association recommends that in patients with controlled blood sugar, at least two $\mathrm{HbA}_{1 \mathrm{c}}$ tests should be conducted once a year, and every 3 months for patients with poor glycemic control. ${ }^{20}$ In this study, only FBG was used to monitor and assess glycemic control. $\mathrm{HbA}_{1 \mathrm{c}}$ testing was not used to monitor or assess glycemic control in any of the patients in the visited health facilities, probably due to the high cost of these tests in the public hospitals.

In this study, the mean age of the T2DM patients was about 55 years, with the majority of them in the age group of 40-59 years. This age group had a higher proportion of patients with poor glycemic control when compared with those in the age group of $<40$ years and $\geq 60$ years. A study conducted in Myanmar by Nyunt et $\mathrm{al}^{21}$ reported that age $\geq 60$ years was associated with poor glycemic control. Other studies have shown that young age is associated with poor glycemic control. ${ }^{22,23}$ The observed variation of association between age and poor glycemic control could be explained by the differences in population characteristics and distribution of age in different studies.

Education of patients, marital status, and employment were not associated with glycemic control in this study. These findings are similar to those reported by other researchers who also did not find an association between glycemic control and low socioeconomic status. ${ }^{24,25}$ Although we did not find a direct association between FBG and factors such as education, employment, and other sociodemographic characteristics, such variables are likely to affect patients' access to quality health care and could therefore have an indirect impact on glycemic control. For instance, educated individuals may have better understanding of, and access to, the type of integrated and comprehensive medical care than those who are less educated and most likely with lesser income to afford medications and the recommended diet for glycemic control.

On the other hand, patients with no health insurance were significantly more likely to have poor glycemic control as compared with patients with health insurance. In this study, only $21.5 \%$ of the patients had insurance for health care. Lack of health insurance affects accessibility and affordability of medicines and diagnostic services in T2DM patients. As a result, lack of health insurance has been linked to low utilization of diabetic preventive services. Interestingly, the study conducted by Harris et $\mathrm{al}^{26}$ did not show an association between health insurance coverage and glycemic control among patients. The observed differences could probably be explained by differences in population characteristics, resource utilizations, human resources, and other support facilities in different settings.

Women in this study were more likely to have poor glycemic control as compared with males. Studies have shown that women have more adverse effects on lipid profile than men, ${ }^{27,28}$ an indication that estrogen-related protective mechanisms may also be affected by diabetes. ${ }^{29}$ The decrease in protective effect of estrogen on body fat distribution and insulin action may also be caused by low-grade inflammation, which may have a greater role in disturbing insulin action in women, or inflammatory factors may interact with female sex hormones. ${ }^{30}$ Although this study did not show significant differences between males and females in terms of their glycemic control, when patients were stratified by age group and sex we found that females in the age group of 40-59 years had significantly higher percentages of poor glycemic control than their male counterparts. This could be due to the fact that in premenopausal women diabetes causes impairment of endothelial function beyond that caused by obesity alone. ${ }^{29}$ The observation that women aged 40-59 years had a higher prevalence of obesity than their male counterparts could probably explain why most women in this age group had poor glycemic control compared with males.

The proportion of patients with poor glycemic control was found to increase with increase in disease duration. A study conducted by Juarez et $\mathrm{al}^{24}$ also showed that patients who had had diabetes for $>10$ years were more than nine times more likely to have poor glycemic control than those who had had diabetes for 3 years. Longer duration of diabetes is known to be associated with poor glycemic control, possibly because of progressive impairment of insulin secretion with time because of the failure of $\beta$-cells, increased insulin resistance to control blood sugar, and eventually decrease in insulin secretion.

In this study, BMI was associated with poor glycemic control. A higher proportion of patients with poor glycemic control was observed in patients with normal BMI, followed by obese and overweight patients. In multivariate analysis, this association between BMI and poor glycemic control remained 
statistically significant, indicating that patients with normal BMI were more likely to have poor glycemic control than overweight patients. This can probably be explained by the fact that patients with poor glycemic control had lost weight due to disease process, while improved glycemic control is associated with weight gain. In addition, obese patients were more likely to have poor glycemic control when compared with overweight patients. This may be due to aggravation of insulin resistance due to increased fat mass and visceral adiposity, which affect insulin sensitivity and cause insulin resistance.

Hypertension and coronary heart diseases were the most frequent comorbidities among T2DM patients in this study. These findings are consistent with the established theory of metabolic syndrome, which is strongly associated with CVD in T2DM. Similar to other studies, no significant differences were found between hypertension and number of comorbidities in patients with poor glycemic control. ${ }^{25}$ The lack of association between hypertension and glycemic control could be explained by the adverse effect of weight gain with improved glycemic control observed in overweight patients, which may worsen other physiological parameters such as hypertension and hypercholesterolemia.

In this study, the majority of T2DM patients were using OHAs, while only $2.1 \%$ of the patients were using a combination of OHA and insulin. A high proportion of patients with poor glycemic control were observed in a group of patients who were using a combination of OHA and insulin. Patients with poor glycemic control require more aggressive treatment with insulin or a combination of $\mathrm{OHA}$ and insulin. Patients who are using OHA alone have good glycemic control when compared with those treated with insulin alone or in combination with OHA. ${ }^{31}$ Lack of an association between the type of treatment and glycemic control in our study could be due to the fact that only a small proportion (14.5\%) of patients were using insulin. Since better glycemic control is achieved through the use of insulin in combination with $\mathrm{OHA}$, it is most likely that the limited use of insulin in this study may have contributed to poor glycemic control among patients. Moreover, the observation that patients who were using a combination therapy of OHA were more than 2.5 times more likely to have poor glycemic control when compared with patients on monotherapy is likely due to the fact that these patients already had an advanced form of diabetes. ${ }^{22}$ This may suggest worsening of diabetes over time and the need for additional medication among patients. Therefore, combination therapy of OHA associated with a higher level of FBG most likely represents a marker of the severity of diabetes rather than the effect of medications. ${ }^{19,20}$
Current guidelines recommend that patients with T2DM should perform at least 150 minutes per week of moderate to intense aerobic exercise, while resistance exercise should be performed at least three times a week. ${ }^{32,33}$ In this study, only a small percentage of patients with T2DM were doing regular physical activity and specific exercise. However, there were no statistically significant differences between patients who did not perform regular physical activity in terms of glycemic control and those who were participating in regular physical exercise. The lack of association between physical exercise and glycemic control in this study is in contrast with the findings by Empierre et al, ${ }^{32}$ probably due to the small number of patients who were performing regular physical activity in this study.

Adherence to antidiabetic medications is crucial to reach metabolic control, since nonadherence is associated with increased levels of $\mathrm{HbA}_{1 \mathrm{c}}$ as well as other negative outcomes such as increased LDL levels, frequent hospitalizations, and mortality. ${ }^{34,35}$ The current study has shown that $38 \%$ of the patients had low adherence to prescribed antidiabetic medications and only $17.4 \%$ of patients could be regarded as adherent to prescribed medications. Patients with poor adherence to prescribed antidiabetic medications had significantly high prevalence of poor glycemic control when compared with those with high and medium medication adherence. Moreover, medication adherence was not associated with complexity of treatment regimen, type of treatment, and duration of diseases. These results are in line with a systematic review of compliance on diabetic treatment that concluded that many T2DM patients comply poorly with treatment, but this is not related to complexity of treatment regimen and severity of the disease. ${ }^{36}$

The high cost of medications was reported by the majority of the patients as the most important reason for nonadherence. The effect of nonadherence to medication due to cost may also be contributed to by the fact that most of the patients in our study had no insurance for health care. Other reasons for nonadherence to medications were forgetfulness, travel, use of alternative medicines, and a sense of feeling well due to decreased diabetic symptoms. These reasons have also been reported in other studies, ${ }^{37,38}$ an indication that more health education is needed to enhance medication adherence and better glycemic control among T2DM patients.

\section{Conclusion}

Overall, the findings from the current study indicate that glycemic control in T2DM patients in the public hospitals 
in Dar es Salaam is generally poor. Independent variables associated with poor glycemic control were lack of insurance for health care, taking more than one OHA, normal BMI, obesity, and low adherence to antidiabetic medications. In order to improve metabolic control, it is recommended that all patients with diabetes should be screened for lipid profile, since high cholesterol, TG, and LDL levels are associated with increased risk of cardiovascular events, and accumulation of cholesterol may contribute to $ß$-cell dysfunction. An education program that emphasizes the importance of medication adherence, physical exercise, and weight management would be of benefit in improving glycemic control among patients.

\section{Acknowledgments}

We acknowledge the financial support provided by the Muhimbili University of Health and Allied Sciences to conduct this study. We thank the management of the Muhimbili National Hospital and medical officers in charge of the municipal hospitals in Dar es Salaam for granting permission and support to conduct the study in the hospitals. We acknowledge the support of medical practitioners and nurses for providing logistical support during interviews, review of patient files, and laboratory assessment of CD4 cell count and lipid profile of patients. We are also grateful to the patients for participating in the study.

\section{Disclosure}

The authors report no conflicts of interest in this work.

\section{References}

1. McLarty D, Kitange H, Mtinangi B. Prevalence of diabetes and impaired glucose tolerance in rural Tanzania. Lancet. 1989;1:871-875.

2. Aspray T, Mugusi F, Rashid S, et al. Rural and urban differences in diabetes prevalence in Tanzania: the role of obesity, physical inactivity and urban living. Trans R Soc Trop Med Hyg. 2000;94:634-644.

3. Chale S, Swai A, Mujinja P, McLarty D. Must diabetes be a fatal disease in Africa? Study of costs of treatment. BMJ. 1992;304:1215-1218.

4. Eriksson J, Fransilla-Kallunki A, Ekstrand A. Early metabolic defects in persons at increased risk for non-insulin-dependent diabetes mellitus. N Engl J Med. 1989;321:690-698.

5. Tripathi BK, Srivastava AK. Diabetes mellitus: complications and therapeutics. Med Sci Monit. 2006;12:RA130-RA147.

6. Girach A, Vignati L. Diabetic microvascular complications: can the presence of one predict the development of another? J Diabetes Complications. 2006;20:228-237.

7. Centers for Disease Control and Prevention. National diabetes fact sheet: general information and national estimates on diabetes in the United States, 2007. Antlanta, GA: US Department of Health and Human Services, Centers for Disease Control and Prevention, 2008.

8. Ciechanowski PS, Katon WJ, Russo JE, Walker EA. The patient-provider relationship: attachement theory and adherence to treatment in diabetes. Am J Psychiatry. 2001;158:29-35.

9. Durán-Varela BR, Rivera-Chavira B, Franco-Gallegos E. [Pharmacological therapy compliance in diabetes]. Salud Publica Mex. 2001;43: 233-236. Spanish.
10. Melikian C, White T, Vanderplas A. Adherence to oral antidiabetic therapy in a managed care organization: a comparison of monotherapy, combination therapy, and fixed-dose combination therapy. Clin Ther. 2002;24:460-467.

11. Hjelm K, Mufunda E, Nambozi G, Kemp J. Preparing nurses to face the pandemic of diabetes mellitus: a literature review. $J$ Adv Nurs. 2003;41:424-434.

12. Rodbard H, Blonde L, Braithwaite S. American Association of Clinical Endocrinologists medical guidelines for clinical practice for the management of diabetes mellitus. Endocr Pract. 2007;13:1-68.

13. Stratton I, Adler A, Neil HA. Association of glycaemia with macrovascular and microvascular complications of type 2 diabetes (UKPDS 35): prospective observational study. BMJ. 2000;321:405-412.

14. Leahy J. Natural history of $\beta$-cell dysfunction in NIDDM. Diabetes Care. 1990;13:992-1010.

15. Norris SL, Lau J, Smith SJ, Schmid CH, Engelgau MM. Self-management education for adults with type 2 diabetes: a meta-analysis of the effect on glycemic control. Diabetes Care. 2002;25:1159-1171.

16. Karter A, Moffet H, Liu J, Parker M. Achieving good glycemic control: initiation of new antihyperglycemic therapies in patients with type 2 diabetes from the Kaiser Permanente Northern California. Am J Manag Care. 2005;11:262-270.

17. Toobert DJ, Hampson SE, Glasgow RE. The Summary of Diabetes Self-Care Measure. Diabetes Care. 2000;23:943-950.

18. Morisky D, Green L, Levine D. Concurrent and predictive validity of a selfreported measure of medication adherence. Med Care. 1986;24:67-74.

19. Nathan DM, Buse JB, Davidson MB, et al. Medical management of hyperglycemia in type 2 diabetes: a consensus algorithm for the initiation and adjustment of therapy: a consensus statement of the American Diabetes Association and the European Association for the Study of Diabetes. Diabetes Care. 2009;32:193-203.

20. American Diabetes Association. Standards of medical care in diabetes. Diabetes Care. 2009;32:S13-S61.

21. Nyunt SW, Howteerakul N, Suwannapong N, Rajatanun T. Self-efficacy, self-care behaviors and glycemic control among type-2 diabetes patients attending two private clinics in Yangon, Myanmar. Southeast Asian $J$ Trop Public Health. 2010;41:943-951.

22. El-Kebbi I, Cook C. Association of younger age with poor glycemic control and obesity in urban African Americans with type 2 diabetes. Arch Intern Med. 2003;163:69-75.

23. Rothenbacher D, Gernot Rüter, Saam S, Brenner H. Younger patients with type 2 diabetes need better glycaemic control: results of a community-based study describing factors associated with a high HbA1c value. Br J Gen Pract. 2003;53:389-391.

24. Juarez DT, Sentell T, Tokumaru S, Goo R, Davis JW, Mau MM. Factors associated with poor glycemic control or wide glycemic variability among diabetes patients in Hawaii, 2006-2009. Prev Chronic Dis. 2012;9:1-10.

25. Haffner S, Hazuda H, Stern M. Effect of socio-economic status on hyperglycemia and retinopathy levels in Mexican Americans with NIDDM. Diabetes Care. 1989;12:128-134.

26. Harris M, Eastman R, Cowie C. Racial and ethnic differences in glycemic control of adults with type 2 diabetes. Diabetes Care. 1999;22:403-408.

27. Howard BV, Cowan LD, Go O, Welty TK, Robbins DC, Lee ET. Adverse effects of diabetes on multiple cardiovascular disease risk factors in women. The Strong Heart Study. Diabetes Care. 1998;21:1258-1265.

28. Juutilainen A, Kortelainen S, Lehto S, Ronnemaa T, Pyorala K, Laakso M. Differences in the impact of type 2 diabetes in coronary heart disease risk. Diabetes Care. 2004;27:2898-2904.

29. Steinberg A, Kortelainen S, Cronin J, Crowde K. Type II diabetes abrogates sex differences in endothelial function in premenopausal women. Circulation. 2000;101:2040-2046.

30. Han TS, Sattar N, Williams K, Gonzalez-Villalpando C, Lean MEJ, Haffner SM. Prospective study of c-reactive protein in relation to the development of diabetes and metabolic syndrome in the Mexico City diabetes study. Diabetes Care. 2002;25:2016-2021. 
31. Turner R. Glycemic control with diet, sulfonylurea, metformin, or insulin in patients with type 2 diabetes mellitus: progressive requirement for multiple therapies (UKPDS 49). JAMA. 1999;281:2005-2012.

32. Umpierre D, Ribeiro PA, Kramer CK, et al. Physical activity advice only or structured exercise training and association with $\mathrm{HbA} 1 \mathrm{c}$ levels in type 2 diabetes: a systematic review and meta-analysis. JAMA. 2011;305:1790-1799.

33. Colberg SR, Sigal RJ, Fernhall B, et al. Exercise and type 2 diabetes: the American College of Sports Medicine and the American Diabetes Association: joint position statement. Diabetes Care. 2010;33: e147-e167.

34. Howteerakul N, Suwannapong N. Adherence to regimens and glycemic control of patients with type 2 diabetes attending a tertiary hospital clinic. Asia Pac J Public Health. 2007;19:43-49.
35. Pladevall M, Williams L, Potts L, Divine G. Clinical outcomes and adherence to medications measured by claims data in patients with diabetes. Diabetes Care. 2004;27:2800-2805.

36. Cramer JA. A systematic review of adherence with medications for diabetes. Diabetes Care. 2004;27:1218-1224.

37. Rhee MK, Cook CB, Dunbar VG, et al. Limited health care access Iimpairs glycemic control in low socio-economic status urban African Americans with type 2 diabetes. $J$ Health Care Poor Underserved. 2005;16:734-746.

38. Wabe NT, Angamo MT, Hussein S. Medication adherence in diabetes mellitus and self management practices among type- 2 diabetics in Ethiopia. N Am J Med Sci. 2011;3:418-423.
Drug, Healthcare and Patient Safety

\section{Publish your work in this journal}

Drug, Healthcare and Patient Safety is an international, peer-reviewed open-access journal exploring patient safety issues in the healthcare continuum from diagnostic and screening interventions through to treatment, drug therapy and surgery. The journal is characterized by the rapid reporting of reviews, original research, clinical, epidemiological and

\section{Dovepress}

post-marketing surveillance studies, risk management, health literacy and educational programs across all areas of healthcare delivery. The manuscript management system is completely online and includes a very quick and fair peer-review system. Visit http://www.dovepress.com/ testimonials.php to read real quotes from published authors.

Submit your manuscript here: http://www.dovepress.com/drug-healthcare-and-patient-safety-journal 